\title{
Ethnic disparities in psychotic experiences explained by area-level syndemic effects
}

Jeremy Coid, Rafael Gonzalez Rodriguez, Constantinos Kallis, Yamin Zhang, Kamaldeep Bhui, Bianca De Stavola, Paul Bebbington and Simone Ullrich

\section{Background}

Ethnic inequalities in health outcomes are often explained by socioeconomic status and concentrated poverty. However, ethnic disparities in psychotic experiences are not completely attenuated by these factors.

\section{Aims}

We investigated whether disparities are better explained by interactions between individual risk factors and place-based clustering of disadvantage, termed a syndemic.

\section{Method}

We performed a cross-sectional survey of 3750 UK men, aged 18-34 years, oversampling Black and minority ethnic (BME) men nationally, together with men residing in London Borough of Hackney. Participants completed questionnaires covering psychiatric symptoms, substance misuse, crime and violence, and risky sexual health behaviours. We included five psychotic experiences and a categorical measure of psychosis based on the Psychosis Screening Questionnaire.

\section{Results}

At national level, more Black men reported psychotic experiences but disparities disappeared following statistical adjustment for social position. However, large disparities for psychotic experiences in Hackney were not attenuated by adjustment for social factors in Black men (adjusted odds ratio, 3.24; $95 \% \mathrm{Cl}$ 2.14-4.91; $P<0.002$ ), but were for South Asian men. A syndemic model of joint effects, adducing a four-component latent variable (psychotic experiences and anxiety, substance dependence, high-risk sexual behaviour and violence and criminality) showed synergy between components and explained persistent disparities in psychotic experiences. A further interaction confirmed area-level effects (Black ethnicity $\times$ Hackney residence, $0.834 ; P<0.001$ ).

\section{Conclusions}

Syndemic effects result in higher rates of non-affective psychosis among BME persons in certain inner-urban settings. Further research should investigate how syndemics raise levels of psychotic experiences and related health conditions in Black men in specific places with multiple deprivations.

\section{Declaration of interest}

K.B. is Editor in Chief of the British Journal of Psychiatry but played no part in the review and decision process.

\section{Keywords}

Psychotic experiences; syndemic; ethnic/racial disparities; area-level effects; multiple morbidity.

\section{Copyright and usage}

(C) The Author(s) 2019. This is an Open Access article, distributed under the terms of the Creative Commons Attribution licence (http://creativecommons.org/licenses/by/4.0/), which permits unrestricted re-use, distribution, and reproduction in any medium, provided the original work is properly cited.
Incidence and prevalence of psychotic phenomena are highest among persons of Black African and Caribbean origin in the $\mathrm{UK}^{1}$ and among African Americans in the USA. ${ }^{2,3}$ To achieve statistical power, incidence studies of ethnic disparities are usually carried out in urban areas where Black and minority ethnic (BME) persons are concentrated and could therefore be influenced by area effects. The highest recorded UK incidence rates were in south and east innerurban London, areas with exceptional socioeconomic deprivation and ethnic density. ${ }^{4,5}$ Prevalence studies have sampled more widely and report similar results. Because psychotic experiences are relatively common in the general population and on a continuum with psychotic symptoms presenting to clinical services, ${ }^{6}$ measuring psychotic experiences in non-clinical samples is important in disentangling individual-level risk factors, such as ethnicity and socioeconomic influences, from population-level area effects.

There are many proposed explanations for ethnic disparities in psychosis. These include migration, racism and discrimination, ethnic density, access to health services, use of cannabis, biological susceptibility, poorer social support and social disadvantage over the life course. ${ }^{7,8}$ Variations have been demonstrated for a range of other health disparities, but these are largely attenuated following statistical adjustments for socioeconomic factors and neighbourhood characteristics. ${ }^{9,10}$ It is then assumed that socioeconomic factors are responsible. However, in the case of psychotic experiences, disparities persist following adjustment for socioeconomic status, ${ }^{2}$ but less is known about the influence of place and area or neighbourhood characteristics.
An alternative and previously untested hypothesis for observed disparities is that area-level effects operate differentially on BME persons in the form of a syndemic, restricted to certain geographical locations where BME persons are concentrated. These are often deprived inner-city areas. It may be that the findings of disparities are a function of place as much as of individual-level risk factors.

\section{Syndemic theory}

A syndemic is defined by Singer et $a l^{11}$ as an aggregation of two or more diseases or other health conditions in a population in which there is some level of deleterious biological or behaviour interface that exacerbates the negative health effects of any or all of the diseases involved. Syndemics involve adverse interaction between diseases and health conditions of all types, including psychiatric morbidity and behavioural conditions, and are most likely to emerge under conditions of health inequality caused by poverty, stigmatisation, stress or structural violence. ${ }^{11}$ These factors show synergy, are mutually causative, and studies need to reconsider how to assess causality. The cumulative effect of experiencing these co-occurring problems is therefore greater than experiencing each constituent problem in isolation. ${ }^{11-13}$

Diseases co-occur in particular temporal or geographical contexts because of harmful social conditions (disease concentration) and can interact at the level of populations and individuals, with 
mutually enhancing deleterious consequences (disease interaction). ${ }^{1-13}$ The first syndemic described consisted of violence, substance misuse and AIDS in poor, minority inner-city populations in the USA, facing multiple political, economic and social challenges of unemployment, substandard housing or homelessness, poor nutrition, disrupted family and social relationships, population displacement and little or no access to healthcare. ${ }^{14}$ Syndemic theory would imply that BME persons living in areas not subject to syndemic effects will not show substantial disparities from the majority White population, and such disparities as emerge will not be attenuated by adjustment for socioeconomic deprivation.

To test this hypothesis, we conducted surveys of young adult men from a representative sample of the UK population and a sample from an area characterised by ethnic and racial diversity with severe socioeconomic deprivation. Our aims were to (a) compare ethnic disparities in the prevalence of psychotic experiences in the general population of the UK with those from a specific deprived area of inner-urban London (BME males compared with the majority White male population); (b) compare BME with White men on a range of mental health and high-risk behaviours; (c) when focussing on psychotic experiences as outcome, investigate whether other health conditions had synergistic effects on their prevalence; and (d) investigate the potential synergistic effects between ethnicity and location on the prevalence of a syndemic comprising four health conditions (including psychotic experiences)

\section{Method}

\section{Study participants}

The Second Men's Modern Lifestyles Survey was carried out in 2011, using random location sampling, an advanced form of quota sampling shown to reduce potential biases introduced when interviewers are able to choose locations to sample from. Individual sampling units (census areas of 150 households) were randomly selected within British regions in proportion to population. The main survey derived a representative sample of young men aged 18-34 years from England, Scotland and Wales. There were two boost surveys: young BME men selected from output areas with a minimum of $5 \%$ BME inhabitants, and output areas in the London Borough of Hackney. Hackney was selected for comparison with the general population because of its ethnic diversity and exceptionally high levels of recorded health and social problems. Identical sampling principles were used for each survey type.

A self-administered questionnaire piloted in a previous survey was adapted. Informed consent was obtained from survey respondents who completed pencil and paper questionnaire in privacy and were paid $£ 5$ for participation (see Supplementary material available at https://doi.org/10.1192/bjp.2019.203). The study was approved by Queen Mary University London Ethics Committee.

The unweighted sample included 3725 men and the weighted sample included 3750 men, all aged 18-34 years; 1999 (53.3\%) were from the representative survey, 991 (26.4\%) were from the BME sample and 760 (20.3\%) were from Hackney. Because most BME persons in the UK are of Black African or Caribbean origin or from the Indian subcontinent (Indian, Pakistani or Bangladeshi - referred to subsequently as South Asian), we excluded other, smaller BME subgroups from analyses.

\section{Health-related and other measures}

We evaluated 19 health-related measures from 4 different domains: sexual health/risks, defined as $\geq 10$ sexual partners in the past year, contraceptive use rare/never, sex with prostitutes, anal sex, sex with men, forced sex on partners or sexually transmitted infection; substance dependence, defined as alcohol or drug dependence; psychiatric morbidity, defined as anxiety disorder, depressive disorder or psychosis; and violence and criminality, defined as repeated assaults/fights, intimate partner violence, fear of violent victimisation, weapon carrying, gang membership, friends encouraging criminal activity, or imprisonment.

The Psychosis Screening Questionnaire (PSQ) ${ }^{15}$ covers hypomania, thought interference, paranoid ideation, strange experiences and auditory and visual hallucinations in the past year. A positive screening for psychosis was recorded if three or more criteria were met, as in previous surveys.

The Hospital Anxiety and Depression Scale ${ }^{16}$ defined anxiety and depression based on scores $\geq 11$ in the past week. Scores $\geq 20$ on the Alcohol Use Disorders Identification Test ${ }^{17}$ and scores $\geq 25$ on the Drug Use Identification Test ${ }^{18}$ were used to identify alcohol or drug dependence, respectively.

Participants were asked if they had ever seen a psychologist or psychiatrist or had ever been admitted to a psychiatric hospital.

\section{Statistical analysis}

Associations between demography and ethnicity were established through logistic regression analyses. Adjusted models were fitted to study relationships between ethnicity and each health outcome, allowing for differential effects by survey. BME subgroups were contrasted with White men in the main survey. Adjustments were made for age, single status, non-UK birthplace and Index of Multiple Deprivation Rank. ${ }^{19}$ We also compared prevalences of health outcomes among ethnic minority groups in the main and Hackney surveys.

To validate the structure of the health domains and derive domain factor scores, we performed confirmatory factor analysis (CFA). A weighted least square mean- and variance-adjusted estimator was used to account for bias in estimates owing to non-linearity of binary indicators. For identification of the CFA model, item variances were allowed to be estimated freely. The model was standardised by fixing factor variances at 1 . Model fit was assessed by root-mean-square error of approximation (RMSEA), the comparative fit index (CFI) and the Tucker-Lewis index (TLI). Values of $\leq 0.06$ for RMSEA, and $\geq 0.95$ for CFI and TLI, indicate very good model fit. ${ }^{20}$ The CFA model showed strong correlations among four health dimensions. To investigate potential syndemic effects, the model was extended to allow for a higher-order latent variable representing a general syndemic dimension, modelled in terms of ethnicity, survey type and their interaction (second-order factor model).

Having used CFA to identify substance misuse, sexual health and violence and crime factors, factor scores derived from this CFA model were then used to examine associations with indicators of psychiatric morbidity (i.e. three or more PSQ criteria and anxiety disorder; prevalence 15\%), and their possible interactions. To avoid issues related to collinearity, we examined associations/interactions using two factors at a time (i.e. pairwise): sexual health with substance misuse, and sexual health with violence and crime.

Appropriate non-response weights were used throughout. We used robust s.e. to account for correlations within survey areas owing to clustering within postcodes. A significance level of 0.05 was adopted throughout.

Statistical analyses were performed using Stata version 13 for Windows and Mplus version 7.1 for Windows (Muthén LK and Muthén BO, Los Angeles, USA; see https://www.statmodel.com/). ${ }^{21}$

\section{Results}

\section{Disparities in psychotic experiences}

BME and White men in Hackney differed in sociodemographic characteristics from White men in the general British population 
(Table 1). Black and South Asian men from the national BME sample were more likely to be born outside the UK and live in areas characterised by multiple deprivation; South Asian men were more likely to be single. In Hackney, White men were more likely to be born outside the UK and fewer were of lower social class and unemployed; Black and South Asian men were more likely to be born outside the UK and fewer were unemployed.

Before adjustment, Black men in the national survey showed a higher mean number of psychotic experiences (particularly thought insertion) than their White counterparts (Supplementary Table 1). After adjustment, no differences were found among Black and Asian men for number of psychotic experiences, one or more psychotic experiences, a categorical classification of psychosis (using PSQ threshold of three or more endorsed items), associated healthcare use including consulting a psychologist/psychiatrist, hospital admission or any of five individual symptoms (Table 2).

However, Black men in Hackney showed multiple differences on all variables, except hallucinations. They were also more likely to have been admitted to a psychiatric hospital. South Asian men in Hackney showed similar trends, although only symptoms of hypomania, thought insertion and hospital admission differed significantly from White men. White men in Hackney were more likely to report thought insertion than Black men.

\section{Disparities in health measures}

Supplementary Figure 1 shows distributions of each health measure among ethnic groups compared with White men in the main national survey. Following adjustments, Black men in the main survey were significantly less likely to report anal sex or sex with men. Only $\geq 10$ sexual partners in the past year was more frequently reported (Table 3). South Asian men in the main national survey were significantly less likely to report sex with prostitutes, anal sex, repeated violence and friends encouraging criminal behaviour. Anxiety disorder was more frequent.

Compared with White men in the main national survey, White men in Hackney were more likely to report $\geq 10$ sexual partners in the past year, alcohol dependence, anxiety disorder and depressive disorder (Table 3). Black men in Hackney were more likely to report a wide range of adverse health measures, including sexually transmitted infections, sex with prostitutes, coercive sex, $\geq 10$ sexual partners, rarely/never using contraceptives, sex with men, alcohol dependence, drug dependence, anxiety disorder, depressive disorder, psychosis, repetitive violence, intimate partner violence, fear of violent victimisation, weapon carrying, gang membership, friends encouraging criminal activity, and imprisonment than White men in the main survey. South Asian men in Hackney were less likely to report anal sex but more likely to report sex with prostitutes, coercive sexual behaviour, $\geq 10$ partners in the past year, rarely/never using contraceptives, alcohol dependence, anxiety disorder, depressive disorder and gang membership than White men in the main survey.

\section{Syndemic model}

We developed a syndemic model, using CFA to include measures of psychiatric morbidity, substance dependence, sexual health and violence and crime. The first step involved identifying first order syndemic factors by domain. The gang membership variable was highly skewed (no White respondents reported gang membership in Hackney) and excluded owing to poor convergence. All items loaded highly except depression and never/rare use of contraceptives. However, these were retained on the basis of clinical relevance. Model fit indices were excellent (RMSEA $=0.025$, 95\% CI $0.022-$ 0.027, $\mathrm{CFI}=0.960$, TLI $=0.952$ ).
Because first-order syndemic factors were highly correlated, we followed standard SEM modelling practice by developing a second-order CFA, thereby generating a general syndemic factor that linked first-order health factor domains. Supplementary Figure 2 confirms that a second-order syndemic factor should be included in the model (fit indices: RMSEA $=0.025,95 \%$ CI 0.022-0.027, CFI $=0.959$, TLI $=0.951$ ).

Table 4 shows evidence of syndemic effect (i.e. positive interaction) between high-risk sexual behaviour and violence/criminality factors in the association with psychotic experiences and anxiety. Similarly, there was evidence of a syndemic effect between the substance misuse and sexual health on the psychotic experiences and anxiety outcome. In the presence of both pairs of factors (substance misuse $\times$ sexual health; sexual health $\times$ violence and criminality), their combined associations with psychotic experiences and anxiety were significantly increased.

Supplementary Table 2 compares BME groups defined by location for the general syndemic factor. In the main national survey, there were no significant differences in factor scores between Black and White men. However, scores for South Asian men were significantly lower $(P<0.001)$ than the White group.

Scores for White, Black and South Asian subgroups in Hackney were significantly higher compared with White men in the main national survey $(P<0.001)$. We estimated the effect of location (i.e. moderation) on ethnic differences in the latent syndemic score. Supplementary Table 2 shows evidence of significant moderation by location on BME group differences from White men. More specifically, being in Hackney significantly increased the latent syndemic score by $0.834(P<0.001)$ among Black men, and by $0.376(P<0.05)$ among South Asian men.

\section{Discussion}

Our findings are new and describe a syndemic of four health conditions: psychotic experiences and anxiety, substance dependence, high-risk sexual behaviour and crime and violence. After selecting psychotic experiences as outcome of interest, we demonstrated synergy between other components on prevalence of men reporting three or more psychotic experiences and severe anxiety. We additionally showed important area-level effects on psychotic experiences. Most significantly, BME in the national population did not show disparities in psychotic experiences observed in previous population studies after adjusting for both social status and neighbourhood effects. This corresponds to US studies of other health outcomes. ${ }^{9,10}$ In addition, they did not report more substance dependence, high-risk sexual behaviour, crime/violence or poorer mental health, with the exception being anxiety disorder among South Asian men. In marked contrast, Black men in Hackney showed higher number of psychotic experiences, more had putative diagnoses of psychosis and all had higher levels of all psychotic experiences, except hallucinations. We also demonstrated an interaction between Black ethnicity and living in Hackney on prevalence of the syndemic (comprising all four health conditions) to indicate location and that Black men were affected most severely by the syndemic.

To the best of our knowledge, the prevalence of Black men in Hackney reporting psychotic experiences was the highest reported among studies using the PSQ. They were also more likely to have consulted a psychiatrist or psychologist and been admitted to a psychiatric hospital. South Asian men were more likely to have experienced thought insertion and hypomania, and to have been admitted to hospital. Anxiety and depressive disorder, alcohol dependence, high-risk sexual behaviour and gang membership were also more prevalent among Black and South Asian men in Hackney, and 
Table 1 Sample characteristics according to participant's ethnicity $(N=3750)$

\begin{tabular}{|c|c|c|c|c|c|c|}
\hline & \multicolumn{3}{|c|}{ Main survey, $N=2990(79.7 \%), n(\%)$} & \multicolumn{3}{|c|}{ Hackney survey, $N=760(20.3 \%), n(\%)$} \\
\hline & White (reference) & Black & Asian & White & Black & Asian \\
\hline All participants & 1795 (60.1) & $492(16.5)$ & 703 (23.5) & 270 (35.5) & $259(34.1)$ & 231 (30.4) \\
\hline \multicolumn{7}{|l|}{ Age, years } \\
\hline $18-24$ & 669 (37.3) & $214(43.6)$ & $273(38.9)$ & 89 (33.1) & $95(36.9)$ & $75(32.6)$ \\
\hline $25-34$ & $1126(62.7)$ & 278 (56.4) & $429(61.1)$ & $181(66.9)$ & $163(63.1)$ & $156(67.4)$ \\
\hline Single & 1045 (58.5) & $326(68.3)$ & $450(66.6)^{*}$ & $177(65.9)^{\star * \star}$ & $143(55.8)$ & $136(59.2)$ \\
\hline Non-UK born & $93(5.2)$ & $103(21.9)^{* * *}$ & $268(31.5)^{\star \star *}$ & $42(16.1)^{\star \star \star}$ & $39(16.0)^{\star \star \star *}$ & $92(42.7)^{\star \star \star}$ \\
\hline \multicolumn{7}{|l|}{ Social class } \\
\hline $\mid$ and $\|^{a}$ & $255(16.9)$ & 48 (13.1) & 104 (19.4) & $59(25.4)$ & $40(19.5)$ & $50(26.4)$ \\
\hline III & $565(37.6)$ & $131(36.2)$ & $152(28.4)$ & $75(32.3)^{*}$ & $71(34.4)$ & $69(36.8)$ \\
\hline IV and V & $388(25.8)$ & $109(30.2)$ & $187(35.0)$ & $58(25.2)^{*}$ & $63(30.6)$ & $57(30.3)^{\star}$ \\
\hline Unemployed & $297(19.7)$ & 74 (20.5) & 93 (17.3) & $40(17.1)^{\star \star \star \star}$ & $32(15.5)^{\star \star}$ & $12(6.5)^{\star \star \star}$ \\
\hline IMDR (mean) & 12370.3 & $7897.9 * * *$ & $8010.0 * * *$ & $4656.2^{\star * \star}$ & $4658.8^{* * *}$ & $4437.3^{* * *}$ \\
\hline
\end{tabular}

Black men reported more violence and criminality. White men in Hackney also reported more anxiety and depression, alcohol dependence and more sexual partners than their counterparts in the general population, showing that all ethnic groups are vulnerable to area effects, and that area $\times$ ethnicity interactions should be studied in future research.

We identified a four-component syndemic that explained our findings. First, we used an aggregation of four health conditions by factor analysis. Second, we examined the interactions between these health conditions, which included substance dependence, high-risk sexual behaviour and violence and criminality on psychotic experiences and anxiety disorder. Although each component represented different domains and pathways, the clustering of multiple risk factors increased the prevalence of psychotic experiences, and vice versa. These analyses correspond to recommendations that multiplicative effects should be demonstrated if the aim is to confirm synergy between hypothesised components of a syndemic. ${ }^{12}$ Third, we examined an additional interaction analysis showing strong area-level effects, which located the syndemic primarily in Hackney and severely affected Black and, to a lesser extent, young South Asian men.

Although social inequality plays a crucial role in the clustering of syndemic factors, ${ }^{23}$ neither social status nor neighbourhood deprivation attenuated the disparities we observed in Hackney. Furthermore, because BME and White subgroups in Hackney were more likely to be employed than their counterparts in the general population, syndemic effects were not simply related to low socioeconomic status.

\section{Ethnic disparities and syndemic effects}

An alternative explanation for previously recorded disparities in psychosis is that syndemic effects differentially affect BME and other socially segregated and excluded subgroups, but in a relatively small number of inner-urban areas characterised by extreme socioeconomic deprivation. These may have disproportionate effects on recorded levels of clinical psychosis in these areas explained by synergy between psychotic experiences and anxiety and severe substance misuse, but also between health-related sexual behaviours and violent/criminal behaviour. The demographic characteristics of Hackney and its level of health problems are seen only in a very small number of inner-urban UK areas. Our findings suggest that BME persons in these areas are unrepresentative of BME persons in national samples and those located elsewhere in the UK, being subject to an excess of risk factors that remain unknown and require further investigation.
The components of the syndemic we identified largely correspond to risk factors previously identified for psychotic experiences, including alcohol and cannabis misuse, ${ }^{24}$ same-sex sexual behaviour, ${ }^{25}$ anxiety disorder, ${ }^{26}$ traumatic experiences and stress, ${ }^{27}$ low social cohesion and crime victimisation. ${ }^{28}$ Bi-directionality between psychotic experiences and risk factors has been specifically demonstrated in alcohol misuse and anxiety disorders (including post-traumatic stress disorder), as well as for psychotic illness. ${ }^{29}$ However, most studies investigate single risk factors which, although strongly associated with psychosis, are not by themselves sufficient to explain a large excess of either psychotic experiences or clinical psychosis in a population.

\section{Pathways and mechanisms}

Further research is required into specific pathways through which the psychiatric morbidity and health conditions we have described interact both in individuals and populations to allow multiplication of adverse health effects. ${ }^{11,12}$ Being exposed to violence, both as victim and perpetrator in violent neighbourhoods, particularly in the context of gang membership, is strongly associated with the specific association between psychotic symptoms and anxiety. ${ }^{30}$ The pathway of this relationship is believed to be stress-promoted immune system deregulation caused by living in a pervasive atmosphere of fear and the perceived threat of ever-present violence. ${ }^{11,30}$

Sexual health and high-risk sexual behaviour correspond to other components of the syndemic. Sexually transmitted diseases, particularly HIV, are associated with anxiety and depression together with substance misuse ${ }^{31}$ and non-sexual violence. ${ }^{32}$ Furthermore, recent research into addictions and addictive behaviours has identified underlying similarities between sexual behaviours (which have features of impulsivity, compulsivity, negative emotional states and craving) and substance use disorders, suggesting common causes ${ }^{32}$ and possible pathways to other psychiatric and cognitive disorders, constituting a basis for dual-diagnosis disorders. ${ }^{33}$ This interpretation fits the notion that syndemic effects lead to illnesses, that these are a manifestation of individual risks and area effects and that multiple disadvantages are pathways to multiple illnesses, which mutually increase risks.

\section{Limitations}

Our study has several limitations. First, our hypothesis that a multicomponent syndemic may explain high rates of psychotic experiences is currently based on results from a single geographical location (Hackney). However, there are a small number of other UK innerurban areas with similar levels of socioeconomic deprivation together 


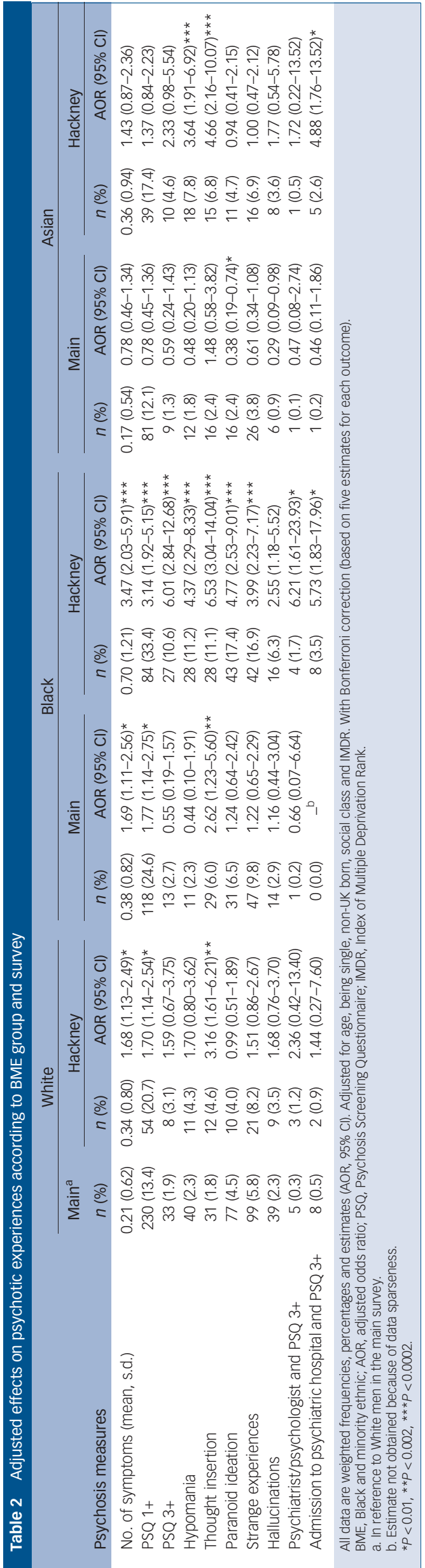

with high levels of ethnic density, in which our findings may be replicated in the future. Furthermore, Hackney was one of three boroughs in east London found to have exceptional incidence rates of psychosis among BME persons a decade earlier. ${ }^{5}$

Although the PSQ assesses only five symptoms, these are central to the diagnosis of psychosis, have good face validity and are likely to be associated with other symptoms in the syndrome of psychosis. It has been the most extensively used instrument to self-report psychotic experiences in UK surveys, with a cut-off of three items shown to have good accuracy for clinical psychosis. ${ }^{34}$

Although we demonstrated synergy between components of the syndemic, these were highly collinear and, in the case of the association between violence/criminality and substance misuse with psychotic experiences and anxiety as outcome, it was not possible to demonstrate an interaction owing to collinearity.

Because White and BME groups in the UK live in closer physical proximity in areas of concentrated poverty than the USA, this may explain why adjustments for socioeconomic deprivation, measured at small area level, did not show the same attenuation between disparities observed in US studies. ${ }^{9,10}$

Our survey was also restricted to young adult men. Previous US studies of syndemics have emphasised the importance of substance misuse, HIV risk behaviour and violent victimisation (substance abuse, violence and AIDS syndemic (SAVA)) among BME women. ${ }^{35}$ However, these have not investigated psychosis. Furthermore, the relationship between violent victimisation reported by these women and high levels of self-reported violent perpetration by men in this study raises the question of whether there is a close association between the two syndemics. This would require further investigation among men in US inner-urban areas where SAVA has been identified in women.

Random location sampling does not provide detailed information on number of young men who declined to participate. However, because the method is based on the National Census, participants were identified and included according to representative strata and their actual frequency in the population. Because young adult men of lower social class are more likely to decline participation in adult household surveys, this method has considerable advantages for investigating health-related behaviours such as violence and criminality, substance misuse and sexual behaviour. The alternative would be to rely on a method requiring statistical weighting to adjust for attrition, which may be particularly high among this subgroup of the population. However, statistical power and effect calculations assume probability sampling and do not technically apply to quota samples like this (see Supplementary material).

\section{Implications}

Our study suggests a new theoretical explanation for ethnic disparities in psychosis observed in UK inner-urban areas. ${ }^{4,5}$ It corresponds to research emphasising social factors in the study of psychosis and urbanicity, indicating effects of social adversity and exclusion in relation to observed geographical variation. Although the study is primarily from the perspective of psychotic experiences as the primary outcome, bi-directionality with anxiety, drug and alcohol misuse, high-risk sexual behaviour and violence and criminality should also be considered, given that each are potential outcomes and equally dependent on each other. The syndemic therefore corresponds to studies from the early 20th century onward, which have reported that these components co-occur and cluster geographically. ${ }^{36,37}$

The prevalence of psychotic experiences originally measured at baseline corresponds to persistence rather than remission of psychotic experiences and later transition to psychotic disorder. ${ }^{38}$ The syndemic we identified could therefore explain both the higher prevalence of psychotic experiences and raised incidence rates of 


\begin{tabular}{|c|c|c|c|c|c|c|}
\hline \multirow[b]{3}{*}{$\begin{array}{l}\text { Health outcomes by } \\
\text { domain }\end{array}$} & \multicolumn{2}{|r|}{ White } & \multicolumn{2}{|c|}{ Black } & \multicolumn{2}{|c|}{ Asian } \\
\hline & Main & Hackney & Main & Hackney & Main & Hackney \\
\hline & & AOR $(95 \% \mathrm{Cl})$ & AOR $(95 \% \mathrm{Cl})$ & AOR $(95 \% \mathrm{Cl})$ & AOR $(95 \% \mathrm{Cl})$ & AOR $(95 \% \mathrm{Cl})$ \\
\hline \multicolumn{7}{|l|}{ Substance misuse } \\
\hline Alcohol dependence & Reference & $4.54(3.00-6.87)^{\star \star * *}$ & $1.98(1.00-3.91)$ & $6.97(4.06-11.96)^{* * *}$ & $1.23(0.59-2.57)$ & $2.89(1.64-5.08)^{\star \star}$ \\
\hline Drug dependence & Reference & $0.34(0.08-1.41)$ & $0.30(0.10-0.88)$ & $12.58(6.26-25.25)^{\star \star \star}$ & $0.59(0.15-2.41)$ & $2.13(0.84-5.38)$ \\
\hline \multicolumn{7}{|l|}{ Mental health } \\
\hline Psychosis (PSQ 3+) & Reference & $1.59(0.67-3.75)$ & $0.55(0.19-1.57)$ & $6.01(2.84-12.68)^{\star \star * *}$ & $0.59(0.24-1.43)$ & $2.33(0.98-5.54)$ \\
\hline Anxiety disorder & Reference & $2.78(1.90-4.06)^{* * *}$ & $1.20(0.69-2.06)$ & $4.92(2.86-8.49)^{* * *}$ & $2.61(1.71-4.00)^{* * *}$ & $3.85(2.57-5.76)^{* * *}$ \\
\hline Depressive disorder & Reference & $3.89(2.46-6.14)^{\star * *}$ & $1.30(0.68-2.50)$ & $4.67(2.86-7.64)^{\star * *}$ & $1.18(0.70-2.00)$ & $5.66(3.42-9.35)^{\star * *}$ \\
\hline \multicolumn{7}{|l|}{ Sexual health } \\
\hline STD & Reference & $1.01(0.61-1.69)$ & $1.11(0.64-1.93)$ & $2.52(1.35-4.71)^{*}$ & $0.35(0.14-0.87)$ & $0.91(0.49-1.70)$ \\
\hline Paid sex worker (ever) & Reference & $1.13(0.62-2.04)$ & $1.00(0.59-1.70)$ & $3.71(2.18-6.29)^{* * *}$ & $0.35(0.16-0.77)^{\star}$ & $2.13(1.28-3.53)^{\star}$ \\
\hline Anal sex & Reference & $1.17(0.79-1.73)$ & $0.39(0.23-0.66)^{\star *}$ & $1.78(1.13-2.80)^{* *}$ & $0.27(0.16-0.47)^{\star * *}$ & $0.43(0.26-0.73)^{* *}$ \\
\hline Coercive sex & Reference & $1.32(0.72-2.41)$ & $0.86(0.37-1.97)$ & $3.60(2.04-6.37)^{\star \star *}$ & $1.33(0.58-3.05)$ & $2.70(1.30-5.62)^{\star}$ \\
\hline $\begin{array}{l}\geq 10 \text { sexual partners (past } \\
\text { year) }\end{array}$ & Reference & $4.96(3.01-8.15)^{* * *}$ & $4.23(2.40-7.46)^{* * *}$ & $7.67(4.64-12.67)^{\star \star *}$ & $0.66(0.24-1.83)$ & $6.18(3.09-12.38)^{\star * *}$ \\
\hline Rare contraceptive use & Reference & $1.24(0.86-1.78)$ & $1.12(0.77-1.62)$ & $2.17(1.41-3.33)^{* *}$ & $1.12(0.81-1.55)$ & $1.96(1.26-3.06)^{*}$ \\
\hline Sexually active MSM & Reference & $1.17(0.57-2.40)$ & $0.19(0.07-0.53)^{* *}$ & $3.67(1.71-7.86)^{* *}$ & $0.68(0.24-1.91)$ & $1.63(0.79-3.36)$ \\
\hline \multicolumn{7}{|l|}{ Violence and criminality } \\
\hline Repeated violence & Reference & $0.52(0.17-1.54)$ & $0.42(0.20-0.90)$ & $3.80(2.08-6.95)^{* * *}$ & $0.16(0.07-0.38)^{* * *}$ & $0.84(0.37-1.91)$ \\
\hline IPV & Reference & $0.33(0.10-1.06)$ & $0.53(0.23-1.23)$ & $7.03(3.85-12.84)^{* \star *}$ & $0.63(0.24-1.62)$ & $1.97(0.90-4.31)$ \\
\hline $\begin{array}{l}\text { Fear of violent } \\
\text { victimisation }\end{array}$ & Reference & $0.99(0.57-1.72)$ & $1.30(0.76-2.20)$ & $3.93(2.31-6.66)^{* * *}$ & $1.64(1.12-2.41)^{\star *}$ & $1.70(1.03-2.82)$ \\
\hline Carried a weapon & Reference & $0.83(0.47-1.50)$ & $0.67(0.34-1.29)$ & $4.74(2.44-9.19)^{* \star *}$ & $0.80(0.35-1.85)$ & $0.93(0.45-1.89)$ \\
\hline Gang membership & Reference & 0 cell & $0.78(0.20-2.94)$ & $42.36(15.81-113.52)^{\star \star * *}$ & $1.35(0.48-3.75)$ & $6.55(2.00-21.46)^{* *}$ \\
\hline Peers encourage crime & Reference & $0.74(0.40-1.35)$ & $0.68(0.42-1.12)$ & $2.53(1.44-4.45)^{\star * *}$ & $0.20(0.11-0.37)^{* * *}$ & $1.96(1.12-3.44)^{*}$ \\
\hline Ever in prison & Reference & $0.57(0.23-1.40)$ & $0.77(0.39-1.53)$ & $2.54(1.19-5.41)^{\star * *}$ & $0.13(0.02-0.81)^{\star * *}$ & $1.00(1.00-1.00)^{\mathrm{a}}$ \\
\hline
\end{tabular}

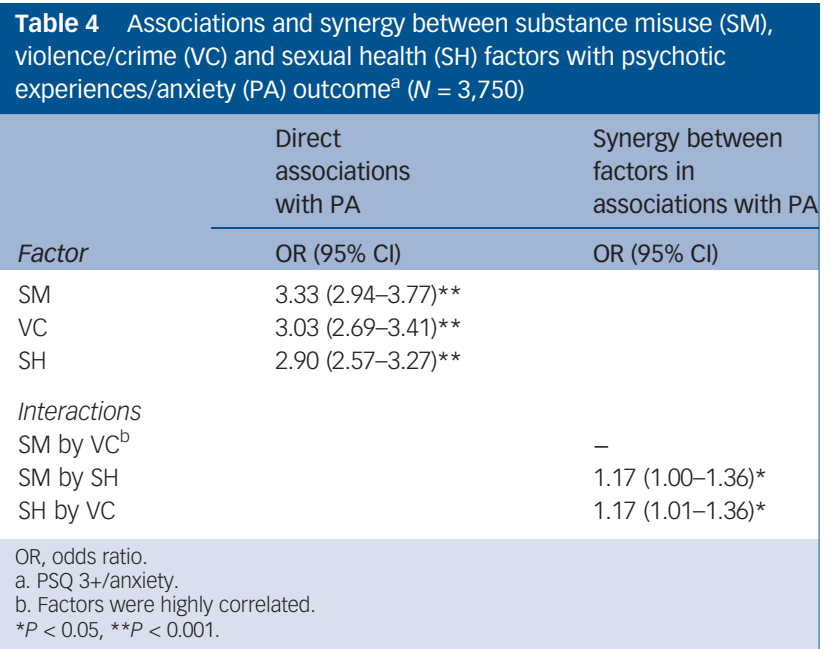

non-affective psychosis among BME persons previously observed in inner-urban areas characterised by concentrated deprivation. ${ }^{1,4,5}$ Our findings also provide a plausible explanation for exceptional levels of demand on clinical and social services in these areas.

Synergy between the four domains of the syndemic may increase the risk of transition to clinical psychosis, representing a significant public mental health problem. This requires further investigation in longitudinal studies. Environmental exposures, particularly stress factors, are believed to interact with genetic risk in modifying biological pathways to psychotic experiences and, with increased stress sensitivity, to schizophrenia. Hyperactivity of the hypothalamicpituitary-adrenal axis and increased sensitivity to stress promote emergence of psychotic experiences. ${ }^{38}$ Transitory developmental expression of psychotic experiences may persist and progress to clinical impairment depending on the degree of environmental risk the person is additionally exposed to. ${ }^{6,38}$ The syndemic effects we identified would therefore imply exceptional risks, further magnified by synergy between these risk factors. However, because these risks are substantially increased by specific health-related behaviours, interventions are needed to discourage progression from psychotic experiences to clinical psychosis by encouraging reduction of these behaviours in vulnerable populations.

A key finding was that most BME men in the UK function as well as their White counterparts despite being more likely to live in lower income households. Young BME men can therefore clearly overcome adverse factors contributing to mental health disparities. However, syndemic effects have a differential impact. It may thus be difficult for BME men to avoid these adverse outcomes when living in areas of concentrated poverty and ethnic density where high-risk behaviours may be condoned and encouraged within socially isolated and excluded population subgroups. Moreover, such behaviours are partly determined by multiple political, economic and social challenges beyond the control of these populations. Interventions that promote mental health equality by reducing the gap between the most and least deprived have been recommended. ${ }^{39}$ However, our findings suggest that additional interventions will be needed to promote behavioural change and healthier lifestyles to avoid risks from the four domains we identified. Improved accessibility and quality of clinical services in these areas through adequate funding are needed. ${ }^{39}$ However, resources are also required to develop new primary prevention strategies, and these may need to target behavioural change to reduce transition to clinical psychosis. A narrow clinical focus on psychotic experiences alone is unlikely to be effective as each component of the syndemic may require specific interventions to reduce transition to clinical psychosis. Finally, we 
require a public mental health approach to prevention and control, not only of the syndemic components, but the forces that originally determined and now tie these components together. ${ }^{11,13}$

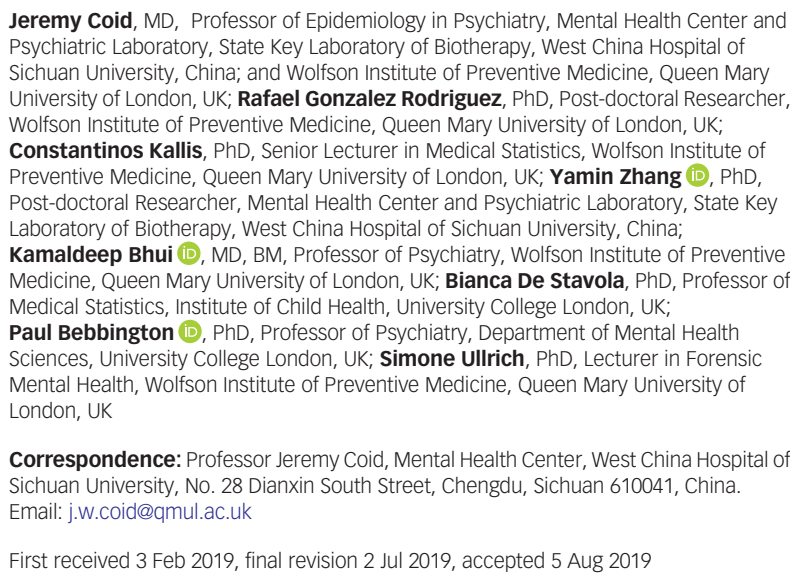

Correspondence: Professor Jeremy Coid, Mental Health Center, West China Hospital of Sichuan University, No. 28 Dianxin South Street, Chengdu, Sichuan 610041, China. Email: j.w.coid@qmul.ac.uk

First received 3 Feb 2019, final revision 2 Jul 2019, accepted 5 Aug 2019

\section{Supplementary material}

Supplementary material is available online at https://doi.org/10.1192/bjp.2019.203.

\section{Funding}

The Maurice \& Jacqueline Bennett Charitable Trust and National Institute of Health Research (NIHR) funded the survey. J.C., C.K., R.G. and S.U. were supported by a programme grant from the NIHR (RP-PG-6407-10500). The contents of this report are solely the responsibility of the authors and do not reflect the official views of the NIHR.

\section{References}

1 Tortelli A, Errazuriz A, Croudace T, Morgan C, Murray RM, Jones PB, et al. Schizophrenia and other psychotic disorders in Caribbean-born migrants and their descendants in England: systematic review and meta-analysis of incidence rates, 1950-2013. Soc Psychiatry Psychiatr Epidemiol 2015; 50(7): 1039-55.

2 Cohen $\mathrm{Cl}$, Marino L. Racial and ethnic differences in the prevalence of psychotic symptoms in the general population. Psychiatr Serv 2013; 64: 1103-9.

3 Bresnahan M, Begg MD, Brown A, Schaefer C, Sohler N, Insel B, et al. Race and risk of schizophrenia in a US birth cohort: another example of health disparity? Int J Epidemiol 2007; 36(4): 751-8.

4 Kirkbride JB, Fearon P, Morgan C, Dazzan P, Morgan K, Tarrant J, et al. Heterogeneity in incidence rates of schizophrenia and other psychotic syndromes: findings from the 3-center AeSOP study. Arch Gen Psychiatry 2006; 63: $250-8$.

5 Coid JW, Kirkbride JB, Barker D, Cowden F, Stamps R, Yang M, et al. Raised incidence rates of all psychoses among migrant groups: findings from the East London first episode psychosis study. Arch Gen Psychiatry 2008; 65(11): 1250-8.

6 Verdoux $\mathrm{H}$, van OS J. Psychotic symptoms in nonclinical populations and the continuum of psychosis. Schizophr Res 2002; 54: 59-65.

7 Gayer-Anderson C, Morgan C. Social networks, support and early psychosis: a systematic review. Epidemiol Psychiatr Sci 2013; 22: 131-46.

8 Veling W, Susser E. Migration and psychotic disorders. Expert Rev Neurother 2011: 11(1): 65-76.

9 Schuster MA, Elliott MN, Kanouse DE, Wallander JL, Tortolero SR, Ratner JA, et al. Racial and ethnic health disparities among fifth-graders in three cities. $N$ Engl J Med 2012; 367(8): 735-45.

10 Adler N, Rehkopf D. U.S. disparities in health: descriptions, causes, and mechanisms. Annu Rev Public Health 2008; 29: 235-52.

11 Singer M, Bulled N, Ostrach B, Mendenhall E. Syndemics and the biosocial conception of health. Lancet 2017; 389: 941-50.

12 Tsai AC, Burns BFO. Syndemics and psychosocial problems and HIV risk: a systematic review of empirical tests of the disease interaction concept. Soc Sci Med 2015; 139: 26-35.
13 Tsai AC, Mendenhall E, Trostle JA, Kawachi I. Co-occurring epidemics, syndemics, and population health. Lancet 2017; 389: 978-82.

14 Wallace R. A synergism of plagues. Environment Research 1988; 47: 1-33.

15 Bebbington PE, Nayani T. The Psychosis Screening Questionnaire. Int J Methods Psychiatr Res 1995; 5: 11-9.

16 Zigmond AS, Snaith RP. The Hospital Anxiety and Depression Scale. Acta Psychiatr Scand 1983; 67: 361-70.

17 Babor TF, Higgings-Briddle JC, Saunders JB, Monteiro M. The Alcohol Use Disorders Identification Test (2nd edn). World Health Organization, 2001.

18 Berman AH, Bergman $\mathrm{H}$, Palmstierna T, Schlyter F. Evaluation of the Drug Use Disorders Identification Test (DUDIT) in criminal justice and detoxification settings and in a Swedish population sample. Eur Addict Res 2005; 11: 22-31.

19 McLennan D, Barnes H, Noble M, Davies J, Garratt E, Dibben C. The English Indices of Deprivation 2010. Department for Communities and Local Government, 2011.

$20 \mathrm{Hu}$ L, Bentler P. Cutoff criteria in fit indexes in covariance structure analysis: conventional criteria versus new alternatives. Struct Equ Modeling 1999; 6(1): $1-55$

21 Muthén L, Muthén B. Mplus User's Guide (7th edn). Muthén \& Muthén, 1998-2013.

22 Office of Population Censuses and Surveys. Standard Occupational Classification: Social Classifications and Coding Methodology Vol 3. Her Majesty's Stationery Office, 1991

23 Oldenburg CE, Perez-Brumer AG, Reisner SL. Poverty matters: contextualizing the syndemic condition of psychological factors and newly diagnosed HIV infection in the United States. AIDS 2014; 28(18): 2763-9.

24 Marconi A, Diforti M, Lewis CM, Murray RM, Vassos E. Meta-analysis of the association between the level of cannabis use and risk of psychosis. Schizophr Bull 2016; 42: 1262-9.

25 Gevonden MJ, Selten JP, Myin-Germeys I, de Graaf R, ten Have M, van Dorsselaer $\mathrm{S}$, et al. Sexual minority status and psychotic symptoms: findings from the Netherlands Mental Health Survey and Incidence Studies (NEMESIS). Psychol Med 2014; 44: 421-33.

26 Hartley S, Barrowclough C, Haddock G. Anxiety and depression in psychosis: a systematic review of associations with positive psychotic symptoms. Acta Psychiatr Scand 2013; 128(5): 327-46.

27 Viechtbauer W, Read I, van Os J. Childhood adversities inverse the risk of psychosis: a meta-analysis of patient-control, prospective and cross-sectional cohort studies. Schizophr Bull 2012; 42: 1903-11.

28 Newbury J, Arsenault L, Caspi A, Moffitt TE, Odgers CL, Fisher HL. Cumulative effects of neighbourhood social adversity and personal crime victimization on adolescent psychotic experiences. Schizophr Bull 2018; 44: 348-58.

29 McGrath JJ, Saha S, Al-Hamzawi A, Andrade L, Benjet C, Bromet EJ, et al. The bidirectional associations between psychotic experiences and DSM-IV mental disorders. Am J Psychiatry 2016; 173: 997-1006.

30 Coid J, Ullrich S, Keers R, Bebbington P, Destavola BL, Kallis C, et al. Gang membership, violence and psychiatric morbidity. Am J Psychiatry 2013; 170 985-93.

31 Rooney BM, Tulloch TG, Blashill AJ. Psychosocial syndemic correlates of sexual compulsivity among men who have sex with men: a meta-analysis. Arch Sex Behav 2018; 47: 75-93.

32 Blum K, Badgaiyan RD, Gold MS. Hypersexuality addiction and withdrawal: phenomenology, neurogenetics and epigenetics. Cureus 2015; 7(10): e348.

33 Chambers RA. Adult hippocampal neurogenesis in the pathogenesis of addiction and dual diagnosis disorders. Drug Alcohol Depend 2013; 130: 1-12.

34 Coid J, Ullrich S. Prisoners with psychosis in England and Wales: diversion to psychiatric inpatient services. Int J Law Psychiatry 2011; 34: 99-108.

35 Singer MC, Erickson PI, Badiane L, Diaz R, Ortiz D, Abraham T, et al. Syndemics, sex and the city: understanding sexually transmitted diseases in social and cultural context. Soc Sci Med 2006; 63(8): 2010-21.

36 Shaw CR, McKay HD. Juvenile Delinquency in Urban Areas. University of Chicago Press, 1942.

37 Faris REL, Dunham HW. Mental IIIness in Urban Areas: An Ecological Study of Schizophrenia and Other Psychoses. University of Chicago Press, 1939.

38 Linscott RJ, Van Os J. An updated and conservative systematic review and meta-analysis of epidemiological evidence on psychotic experiences in children and adults: on the pathway from proneness to persistence to dimensional expression across mental disorders. Psychol Med 2013; 43: 1133-49.

39 Marmot M, Friel S, Bell R, Houweling TA, Taylor S. Closing the gap in a generation: health equity through action on the social determinants of health. Lancet 2008: 372(9650): 1661-9. 